\title{
Effects of the acute exposure to the electromagnetic field of mobile phones on human auditory brainstem responses
}

Received: 14 November 2003/ Accepted: 3 November 2004 / Published online: 25 February 2005

(C) Springer-Verlag 2005

\begin{abstract}
The purpose of this study was to evaluate the short-term effects of the electromagnetic fields (EMF) of mobile phones on human auditory brainstem responses. This prospective study of healthy adults evaluated the influence of EMF. Eighteen healthy adult volunteers participated in this study. Mobile telephones emitting signals in the region of $900 \mathrm{MHz}$ and with the highest SAR value of $0.82 \mathrm{~W} / \mathrm{kg}$ were positioned in direct contact to the right ear, which was exposed to the phone signal for $15 \mathrm{~min}$ before and after ABR testing with click stimuli of 60 and $80 \mathrm{~dB}$ $\mathrm{nHL}$ intensities. The latencies of the waves and interwave latencies were measured on screen by an experienced audiologist. The differences of the mean latencies of waves I, III and IV were not significant in initial and post-exposure ABR measurements at both 60 and $80 \mathrm{~dB}$ nHL stimulus levels $(P>0.05)$. Similarly, differences of the mean interwave intervals I-III, $\mathrm{I}-\mathrm{V}$ and III-V remained insignificant at the initial and postexposure ABR measurements at stimulus levels of both 60 and $80 \mathrm{~dB}$ nHL $(P>0.05)$. Acute exposure to the EMF of mobile phones does not cause perturbations in ABR latencies. However, these negative results should not encourage excessive mobile communication, because minor biological and neurophysiological influences may not be detectable by the current technology.
\end{abstract}

\footnotetext{
C. Oysu

Department of Otolaryngology,

Haydarpasa Numune State Hospital, Istanbul, Turkey

M. Topak · O. Celik · H. B. Yilmaz · A. A. Sahin

Department of Otolaryngology,

Taksim State Hospital, Istanbul, Turkey

C. Oysu ( $\square)$

Haci Hakki Bey sk. Basel ap. No:4/7, Erenkoy,

34728 Istanbul, Turkey

E-mail: coysu@doruk.net.tr

Tel.: + 90-216-4671109

Fax: + 90-212-2445541
}

Keywords Cellular phone radiation · Auditory brainstem responses · Electromagnetic field - Biological effects

\section{Introduction}

The introduction of mobile phones into daily life has caused public concerns regarding the possible adverse effects of electromagnetic radiation on human health. Heating of biological tissues is a consequence of the thermal effects of electromagnetic fields (EMF), but studies have shown that this is not a major health issue for most handsets that do not violate safety guidelines [1]. Still, the non-thermal effects of EMF on living organisms are a subject of research. Because of the proximity of mobile phone handsets to the head, the central nervous system (CNS) is exposed to higher specific absorption rates (SAR) of $\sim 900$ or $1,800 \mathrm{MHz}$ of microwave radiation transmitted by the device [2]. This fact has led several researchers to investigate the non-thermal biological effects of microwave radiation on CNS.

Although opposing studies exist [3, 4], several studies have reported the effects of EMF on EEG [5, 6, 7]. Reiser et al. [6] reported that $15 \mathrm{~min}$ of exposure to $900 \mathrm{MHz}$ pulsed microwave radiation results in an increase in beta-1 and delta-power on EEG. Von Klitzing [7] found changes in the alpha activity pattern on EEG immediately after exposure. In two recent studies, Krause et al. $[5,8]$ reported that exposure to EMF modulates EEG responses specifically during cognitive processes and auditory memory tasks.

Our knowledge regarding the influences of EMF on the auditory system is based mainly on animal studies [9, $10,11,12$ ]. Chou et al. [3] reported that exposure to 918$\mathrm{MHz}$ pulsed microwave is accompanied by a mechanical disturbance of the hair cells in the guinea pig cochlea. Later on, the same authors reported that EMF influences evoked auditory potentials in guinea-pigs and rats $[10,14]$. But the effects of EMF exposure to the human 
auditory system remained uninvestigated until very recently [15]. Ozturan et al. [15] reported no measurable changes in otoacoustic emissions following $10 \mathrm{~min}$ of exposure. Although this study shows clearly that the EMF of mobile phones has no effect at the cochlear level, the influence on the rest of the auditory system is obscure. The aim of the current study is to investigate the acute effects of EMF in the short term on healthy human auditory brainstem responses (ABR).

\section{Material and methods}

Subjects

Eighteen healthy right-handed adult volunteers (11 males and 7 female; mean age, 23 years; range, 20 to 28) participated in this study. All subjects had bilateral puretone thresholds of $15 \mathrm{~dB}$ or better in frequencies from 250 to $8,000 \mathrm{~Hz}$ and had normal type A tympanograms. Volunteers with tinnitus, middle ear pathology or a history of noise exposure were not enrolled in the study. All subjects gave their written informed consent prior the experiment. The Medical Ethical Review Committee of Taksim State Hospital approved the study.

\section{EMF procedure}

A Nokia 6310i mobile telephone was positioned in direct contact to the right ear as in normal communication. This mobile telephone emits and receives radio signals in the region of $900 \mathrm{MHz}$, and the highest SAR value for this model when tested for compliance against the standard was $0.82 \mathrm{~W} / \mathrm{kg}$. After the measurement of initial ABR, without removing the electrodes, the subject's ear was exposed to the activated mobile phone signal for $15 \mathrm{~min}$. Then the mobile device was turned off, and ABR testing was repeated.

\section{ABR measurements}

The auditory brainstem response was recorded with the Interacoustics EP15 computerized ABR system (Assens, Denmark) running Windows 98. Active electrodes were attached to the ipsilateral mastoid region and were referenced to a vertex electrode. Click acoustic stimuli, alternating in polarity, were presented by an earphone on the ear at a rate of $21 / \mathrm{s}$ with 60 and $80 \mathrm{~dB} \mathrm{nHL}$ intensities. With a filter setting of 100 to $3,000 \mathrm{~Hz}, 1,500$ sweeps were averaged. The latencies of the waves were measured with a cursor from a screen by an experienced audiologist.

Statistical method

Statistical analysis was performed using GraphPad Prisma V.3 statistical software. Continuous variables expressed as mean and standard deviations were compared using the paired $t$-test. A probability value less than 0.05 was regarded as significant.

\section{Results}

At the initial ABR measurement at $60 \mathrm{~dB}$ nHL, the stimulus level mean latencies of waves I, III and V were $2.12 \mathrm{~ms}$ (SD: 0.14), $4.11 \mathrm{~ms}$ (SD: 0.27) and $5.94 \mathrm{~ms}$ (SD: 0.35 ), respectively. Following acute exposure to the EMF of the mobile phone, the mean latencies were $2.17 \mathrm{~ms}$ (SD: 0.16) for wave I, $4.10 \mathrm{~ms}$ (SD: 0.23) for wave III and $5.97 \mathrm{~ms}$ (SD: 0.33) for wave V, and the differences of the initial and postexposure latencies were not significant ( $P>0.05$; Fig. 1$)$.

At $80 \mathrm{~dB} \mathrm{nHL}$, the click stimulus level initial latency means for waves I, III and V were $1.41 \mathrm{~ms}$ (SD: 0.14), $3.62 \mathrm{~ms}$ (SD: 0.34) and $5.48 \mathrm{~ms}$ (SD: 0.30), respectively. Post-exposure latency means were $1.41 \mathrm{~ms}$ (SD: 0.12) for wave I, $3.60 \mathrm{~ms}$ (SD: 0.19) for wave III and $5.48 \mathrm{~ms}$ (SD: 0.29 ) for wave $\mathrm{V}$, and the differences of the initial and succeeding latencies were not significant $(P>0.05$; Fig. 2).

Mean interwave intervals (I-III, I-V and III-V) were $2.02 \mathrm{~ms}$ (SD: 0.24) for the first, $3.88 \mathrm{~ms}$ (SD: 0.34) for the second and $1.79 \mathrm{~ms}$ (SD: 0.27) for the last interval at baseline ABR study with $60 \mathrm{~dB}$ nHL stimulus. Following EMF exposure, the mean intervals for waves I-III, I$\mathrm{V}$ and III-V were $2.00 \mathrm{~ms}$ (SD: 0.18), $3.93 \mathrm{~ms}$ (SD: 0.28) and $1.82 \mathrm{~ms}$ (SD: 0.25), respectively, and the differences between initial and postexposure intervals were not significant $(P>0.05)$.

Similar baseline measurements were done at $80 \mathrm{~dB}$ nHL stimulus level mean intervals for I-III, I-V and IIIV $2.21 \mathrm{~ms}$ (SD: 0.16); $3.98 \mathrm{~ms}$ (SD: 0.27) and $1.80 \mathrm{~ms}$ (SD: 0.19) were calculated, respectively. Following EMF exposure the mean intervals for waves I-III, I-V and III$\mathrm{V}$ were $2.20 \mathrm{~ms}$ (SD: 0.15), $4.06 \mathrm{~ms}$ (SD: 0.29) and $1.83 \mathrm{~ms}$ (SD: 0.24$)$, respectively, and the differences of

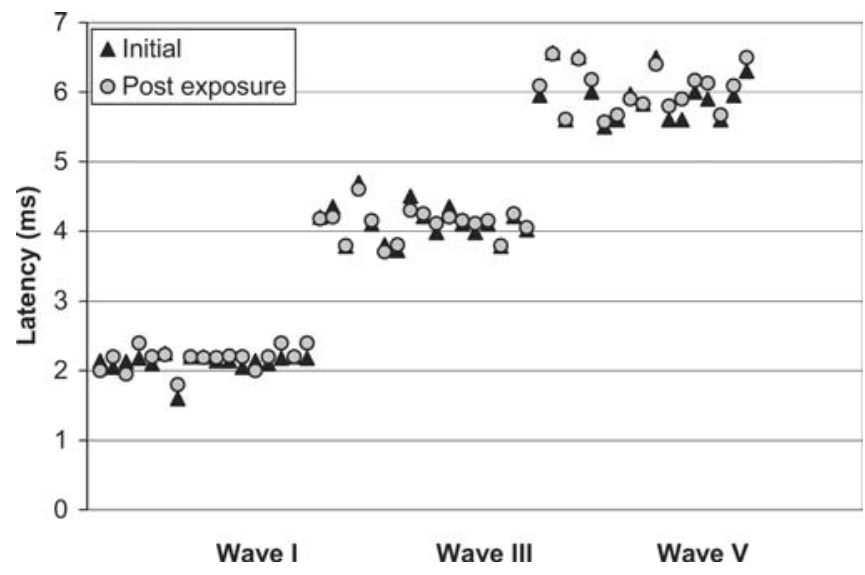

Fig. 1 Scatter plot displaying the initial and post exposure latencies of three waves at $60-\mathrm{dB}$ stimulus level in 18 subjects 


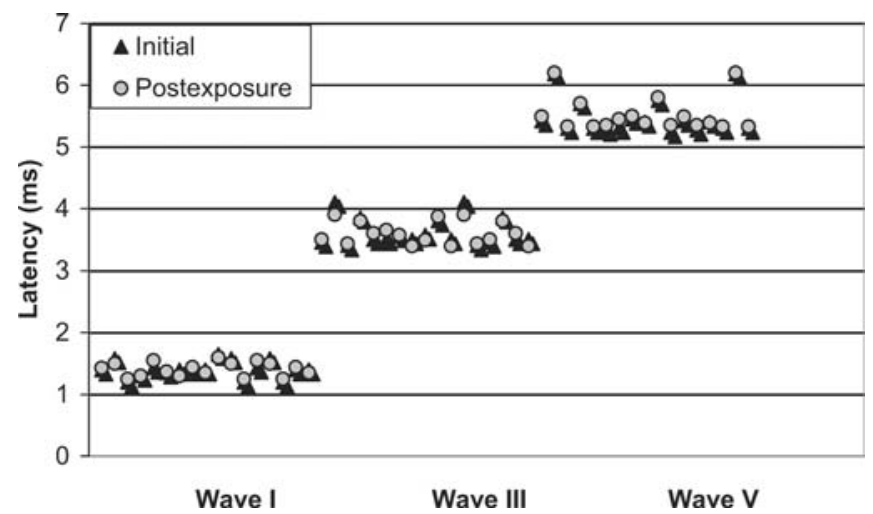

Fig. 2 Scatter plot displaying the initial and post exposure latencies of three waves at $80-\mathrm{dB}$ stimulus level in 18 subjects

the initial and post-exposure interval means were not significant $(P>0.05)$.

\section{Discussion}

The use of cellular mobile phones has increased dramatically in recent years. In Turkey, approximately one in every three people owns a mobile phone, and the average monthly communication time for users is 40 min per month. There is a constant rise in these numbers, which is even more accentuated in western communities [16].

The dependence on mobile communication and its possible adverse effects raises public anxiety. The biological effects of the EMF emitted by mobile phones are generally grouped as thermal and non-thermal. Modern mobile phones may raise the temperature of deep tissues by maximally $0.1^{\circ} \mathrm{C}$ [17]. Nevertheless, the upper limit of temperature increase assumed to be non-detrimental for human health is $1^{\circ} \mathrm{C}[17,18]$. However, several thermal effects of EMF on living organisms are shown in experimental studies, such as an alteration of the permeability of the blood-brain barrier [19]. Modification of sleep patterns [20], increase in blood pressure [21], potential genotoxicity and DNA strand breaks [22] are among the non-thermal effects of EMF. Regarding these biological changes, probably there is no clear cut between the thermal and non-thermal effects of EMF; a combination of both may be responsible.

Widespread use of handsets instead of hand-free devices makes the brain vulnerable to the effects mentioned above. Acute exposure of rats to amplitude-modulated microwaves resulted in changes of energy metabolism in the brain as a consequence of direct disturbance of the mitochondrial electron transport [23]. Moreover, an alteration of the neurotransmitter activities, such as a fluctuation in acetylcholinesterase activity or fall in sodium-dependent high-affinity choline uptake, is reported in mammals $[24,25,26]$. Probably, these metabolic, electrochemical and other unknown effects of EMF lead to alterations in biological electrical activities. In animal studies, an increase in total EEG spectral power or delta power is shown following EMF exposure [27]. As an electrochemical instrument, the human brain must also be influenced by EMF. Although universally no conclusions could be reached so far, several alterations of human EEG by EMF have been reported [5, 28, 29, 30]. The main finding in these human EEG studies is the decrease in sleep onset latency and the enhancement in superficial sleep stages [20]. Modulation in the response of EEG oscillatory activity during the cognitive process is another observed influence of EMF on brain activity. Recently, Krause et al. [8] reported that exposure to EMF causes some desynchronization on EEG during auditory memory tasks.

As a part of CNS, one could not assume an auditory apparatus immune from these effects of EMF. An exponential increase in SAR is shown as the distance from the mobile phone decreases, or vice versa [30]. Therefore, auditory pathways must be as prone to the effects of EMF as other regions of the brain. In fact, animal studies done with evoked-response audiometry (ERA) showed that evoked responses might be elicited by microwaves [11, 14]. Furthermore, Seaman and Lebovitz [9] showed that response characteristics of the cochlear nucleus to microwave pulses were similar to the acoustic stimuli in cats. These and some other animal studies have demonstrated that EMF may severely influence auditory functions or test results. It is reasonable to expect that the EMF of mobile phones modulates the electrical activity of the auditory system like the brain. On the other hand, our current knowledge regarding the effects of mobile phones on the human auditory system is mainly limited to the recent work of Ozturan et al. [15]. In this study, the authors concluded that $10 \mathrm{~min}$ of exposure to the EMF emitted from mobile phones had no effect at the cochlear level. But the effects of EMF emitted from mobile phones to the rest of the auditory system or, in other words, auditory tests such as ABR, have not been investigated so far.

In this study, we investigated in healthy adults the effects of EMF transmitted by mobile cellular phones to ABR, which represents the electrical activity of the distal portion of the auditory pathway with five waveforms. We compared intrasubject changes in absolute latencies and intervals of waves I, III and V, representing the electrical activity of the cochlear nerve, cochlear nucleus and lateral lemniscus, respectively, following $15 \mathrm{~min}$ of EMF exposure [31]. Our study revealed that EMF causes no significant alteration in the latencies of these three major waves obtained at both 60 and $80 \mathrm{~dB}$ nHL stimulus levels. Similarly, the changes in I-III, I-V and III-V interweave intervals remain insignificant following EMF exposures at both stimulus levels. All these data show that acute short-term exposure to EMF does not alter auditory nerve electrical conduction.

Although focused on a different level of the human auditory system, our study concurs with the results of Ozturan et al. [15]. The current study demonstrated that 15-min exposure to EMF emitted by mobile phones has no influence on ABR. Three major possible explanations 
may be made for this negative result. First, longer exposures such as $60 \mathrm{~min}$ may have altered these results, but such communication time is not preferred because it is beyond the monthly average in most countries. Second, modern mobile phones have no actual effect on the human auditory system. Because SAR decreases exponentially as the distance from the EMF source increases, the brainstem may be under less influence than the temporal lobe [30]. Also, the brainstem may be assumed to be protected by the thicker petrous part of the temporal bone. Third, EMF may have minor influences that cannot be detected by current ABR technology. Our $A B R$ recordings were made after the termination of mobile phone communication because EMF influences both the stimulus source and the recording system negatively. Although some experimental methods measuring otoacoustic emissions simultaneously exist, currently, there is no worldwide available procedure to record ABR simultaneously with mobile phone communication. Moreover, ABR recordings made at 60 and $80 \mathrm{~dB}$ stimulus levels may not reflect the spontaneous electrical activity of the auditory pathway. It can be speculated that these click stimuli may mask the effect of EMF on the brainstem electrical activity.

This study shows merely that the acute EMF of mobile phones do not cause perturbations in ABR latencies in the short term. Together with previous otoacoustic emission studies, it can be concluded that EMF do not influence human hearing functions in the short term [15]. However, the observed negative results should not encourage unnecessary and excessive mobile communication, because minor biological and neurophysiological influences may be beyond the capabilities of our current recording technology. Neither the current study nor the body of literature provides sufficient information regarding the long-term biological effects of EMF. Concerns regarding the adverse effects of EMF on human health are lasting; therefore, guidelines for limiting exposure to electromagnetic fields should be followed [32].

\section{References}

1. Gandhi OP, Lazzi G, Furse CM (1996) Electromagnetic absorption in the human head and neck for mobile telephones at 835 and 1,900 MHz. IEEE transactions on microwave theory and techniques 44:1884-1897

2. Schonborn F, Burkhardt M, Kuster N (1998) Differences in energy absorption between heads of adults and children in the near field of sources. Health Phys 74:160-168

3. Roschke J, Mann K (1997) No short-term effects of digital mobile radio telephone on the awake human electroencephalogram. Bioelectromagnetics 18:172-176

4. Wagner P, Roschke J, Mann K, Hiller W, Frank C (1998) Human sleep under the influence of pulsed radiofrequency electromagnetic fields: a polysomnographic study using standardized conditions. Bioelectromagnetics 19:199-202

5. Krause CM, Sillanmaki L, Koivisto M, Haggqvist A, Saarela C, Revonsuo A, Laine M, Hamalainen H (2000) Effects of electromagnetic fields emitted by cellular phones on the electroencephalogram during a visual working memory task. Int J Radiat Biol 76:1659-1667
6. Reiser H, Dimpfel W, Schober F (1995) The influence of electromagnetic fields on human brain activity. Eur J Med Res $1: 27-32$

7. von Klitzing L (1995) Low-frequency pulsed electromagnetic fields influence EEG of man. Phys Med 11:77-80

8. Krause CM, Sillanmaki L, Koivisto M, Haggqvist A, Saarela C, Revonsuo A, Laine M, Hamalainen H (2000) Effects of electromagnetic field emitted by cellular phones on the EEG during a memory task. Neuroreport 11:761-764

9. Seaman RL, Lebovitz RM (1989) Thresholds of cat cochlear nucleus neurons to microwave pulses. Bioelectromagnetics 10:147-160

10. Chou CK, Guy AW (1979) Microwave induced auditory responses in guinea pigs: relationship of threshold and microwave-pulse duration. Radio Sci 14:193-197

11. Chou CK, Guy AW, Galambos R (1982) Auditory perception of radiofrequency electromagnetic fields. J Acoust Soc Am 71:1321-1334

12. Taylor EM, Ashleman BT (1974) Analysis of central nervous system involvement in the microwave auditory effect. Brain Res 74:201-208

13. Chou C, Galambos R, Guy AW, Lovely RH (1975) Cochlear microphonics generated by microwave pulses. J Microw Power 10:361-367

14. Chou CK, Yee KC, Guy AW (1985) Auditory response in rats exposed to $2,450 \mathrm{MHz}$ electromagnetic fields in a circularly polarized waveguide. Bioelectromagnetics 6:323-326

15. Ozturan O, Erdem T, Miman MC, Kalcioglu MT, Oncel S (2002) Effects of the electromagnetic field of mobile telephones on hearing. Acta Otolaryngol 122:289-293

16. Harma M (2000) Electric hypersensitivity and neurophysiological effects of cellular phones - facts or needless anxiety? Scand J Work Environ Health 26:85-86

17. Van Leeuwen GM, Lagendijk JJ, Van Leersum BJ, Zwamborn AP, Hornsleth SN, Kotte AN (1999) Calculation of change in brain temperatures due to exposure to a mobile phone. Phys Med Biol 44:2367-2379

18. UNEP/WHO/IRPA. Electromagnetic fields $(300 \mathrm{~Hz}$ to $300 \mathrm{GHz}$ ): Environmental Health Criteria 137. World Health Organization, Geneva

19. Fritze K, Sommer C, Schmitz B, Mies G, Hossmann KA, Kiessling M, Wiessner C (1997) Effect of global system for mobile communication (GSM) microwave exposure on bloodbrain barrier permeability in rat. Acta Neuropathol (Berl) 94:465-470

20. Mann K, Roschke J (1996) Effects of pulsed high-frequency electromagnetic fields on human sleep. Neuropsychobiology 33:41-47

21. Braune S, Wrocklage C, Raczek J, Gailus T, Lucking $\mathrm{CH}$ (1998) Resting blood pressure increase during exposure to a radio-frequency electromagnetic field. Lancet 351(9119):18571858

22. Lai H, Singh NP (1996) Single- and double-strand DNA breaks in rat brain cells after acute exposure to radiofrequency electromagnetic radiation. Int J Radiat Biol 69:513-521

23. Sanders AP, Joines WT (1984) The effects of hyperthermia and hyperthermia plus microwaves on rat brain energy metabolism. Bioelectromagnetics 5:63-70

24. Baranski S (1972) Histological and histochemical effect of microwave irradiation on the central nervous system of rabbits and guinea pigs. Am J Phys Med 51:182-191

25. Dutta SK, Das K, Ghosh B, Blackman CF (1992) Dose dependence of acetylcholinesterase activity in neuroblastoma cells exposed to modulated radio-frequency electromagnetic radiation. Bioelectromagnetics 13:317-322

26. Lai H, Carino MA, Horita A, Guy AW (1992) Opioid receptor subtypes that mediate a microwave-induced decrease in central cholinergic activity in the rat. Bioelectromagnetics 13:237-246

27. Thuroczy G, Kubinyi G, Bodo M, Bakos J, Szabo LD (1994) Simultaneous response of brain electrical activity (EEG) and cerebral circulation (REG) to microwave exposure in rats. Rev Environ Health 10:135-148 
28. Hermann DM, Hossmann KA (1997) Neurological effects of microwave exposure related to mobile communication. $\mathbf{J}$ Neurol Sci 152:1-14

29. Freude G, Ullsperger P, Eggert S, Ruppe I (1998) Effects of microwaves emitted by cellular phones on human slow brain potentials. Bioelectromagnetics 19:384-387

30. Freude G, Ullsperger P, Eggert S, Ruppe I (2000) Microwaves emitted by cellular telephones affect human slow brain potentials. Eur J Appl Physiol 81:18-27
31. Moller AR, Jannetta PJ (1983) Interpretation of brainstem auditory evoked potentials: results from intracranial recordings in humans. Scand Audiol 12:125-133

32. International Commission on Non-Ionizing Radiation Protection (1998) Guidelines for limiting exposure to time-varying electric, magnetic and electromagnetic fields (up to $300 \mathrm{GHz}$ ). Health Phys 74:494-522 